\title{
THEORETICAL INTERPRETATION OF THE HERTZSPRUNG-RUSSELL DIAGRAM
}

\author{
(Introductory Paper) \\ M. SCHWARZSCHILD \\ Princeton University Observatory
}

I N T R O D U C T I O N

The theory of the stellar interior indicates that for a star of given mass, given initial composition, and given age, all physical characteristics (including the luminosity and the effective temperature) are uniquely determined. Accordingly it should be possible to derive theoretically for a particular star of definite mass and initial composition a relation between luminosity and effective temperature, i.e., a curve in the Hertzsprung-Russell diagram along which the star must move during its evolution. With the help of such evolution curves it must then be possible to identify any observed star as to its mass, composition and age. This identification is possible at present only for certain stellar types, for two reasons. First, the theoreticians have not yet succeeded in deriving all the necessary evolution curves. Second, the evolution curves cross over each other in a complicated pattern in the Hertzsprung-Russell diagram so that observations in the two dimensional Hertzsprung-Russell diagram will not always suffice to identify uniquely the state of an individual star although it appears to suffice for the identification of a whole sequence of stars, like those which are observed in stellar clusters.

The following paragraphs give a short review of those phases of stellar evolution which at present can be identified with observed types of stars with fair certainty. Many of the problems here touched upon will be more thoroughly discussed in the subsequent communications of this symposium.

\section{P RE - M A I N - S E Q U E N C E CONTRACTION}

A star enters the Hertzsprung-Russell diagram during its contraction from an interstellar cloud. During the later portion of this contraction the stellar luminosity will vary only little while the radius steadily shrinks, i.e. the effective temperature steadily increases. This pre-mainsequence phase has recently been identified with the relatively faint red stars in very young clusters and associations. It appears plausible that in the last phases of this contraction rotational instability may cause mass ejection which might explain the well known spectroscopic peculiarities of the $\mathrm{T}$ Tauri stars.

In connection with this early evolutionary phase a problem exists as to whether there might occur, in the solar neighborhood, a very large number of faint infrared stars. Owing to their low mass and luminosity these could evolve so slowly that even the full galactic time scale would not suffice to bring them into the more easily observable portion of the HertzsprungRussell diagram. 
The initial main-sequence state is theoretically defined as that state in which the star first burns nuclear fuel. Models for this state have been derived for stellar masses from about onetenth of a solar mass to over a hundred solar masses. These models are homogeneous in composition and not dissimilar in overall structure to the classical standard model. Stars of more than two solar masses have, in this state, convective cores and radiative envelopes, but the reverse is true for less massive stars.

The theoretical initial main-sequence state can be identified with the observed initial main sequence recently derived from a combination of galactic clusters. The majority of the high-velocity sub-dwarfs can probably be identified with the same state, their difference from the regular dwarfs being caused probably by their much lower heavy element abundance.

\section{A I N-SE Q U E N C E B A N D}

The early phases of evolution may be defined as those phases in which the hydrogen content at the center of the star is depleted from its initial value (corresponding to the initial mainsequence state) to zero. These phases have been computed in detail for a number of stellar masses ranging from one solar mass to a hundred solar masses. Even though the internal developments during these phases differ noticeably from one mass range to another, the corresponding evolution tracks in the Hertzsprung-Russell diagram appear to have for all masses the same general character : - the luminosity steadily rises and so does the radius, so that the evolution track moves from the initial main sequence upwards and to the right, reaching a distance from the initial main sequence of $0 . I$, in the logarithm of the effective temperature for the lower masses, and about 0.2 for the highest masses.

It is clear that these early evolution phases must be identified with the main sequence band which is the most populated feature of the observed Hertzsprung-Russell diagram.

The theoretical computations show that the less massive stars during the early evolution phases burn only about one-eighth of their total store of hydrogen while the most massive stars burn up more than one-half of their hydrogen. In spite of this higher total consumption the massive stars spend, because of their high rate of consumption, a shorter time in the mainsequence phases than the less massive stars; a star of a hundred solar masses has a mainsequence lifetime of about three million years while a star of one solar mass has one of approximately ten billion years. This great variation of main-sequence lifetime with mass has one important consequence; all stars of spectral types $\mathrm{A}_{5}$ and earlier have main-sequence lifetimes substantially shorter than the probable galactic lifetime, whereas stars of spectral type $\mathrm{F}_{7}$ and later have main-sequence lifetimes substantially longer than the galactic life. This astrophysical circumstance, therefore, divides the main sequence fairly sharply into two sections which must show very different characteristics in stellar statistical investigations.

\section{R E D G I A N T S}

The theoretical evolution tracks derived for the advanced phases following the exhaustion of hydrogen at the stellar center are as yet few and approximate. They indicate that these phases are characterized by a steadily accelerating contraction of the core and a simultaneous 
expansion of the envelope. Clearly these phases must be identified with the red giants and subgiants.

In the less massive stars the core contraction leads to steadily increasing degeneracy and only in the latest phases to an appreciable heating of the core, while at the same time the envelope becomes convective. These processes cause a smooth rise of luminosity and radius and appear to represent satisfactorily the continuous red-giant branches in the globular clusters and in the oldest galactic clusters. Here again the difference in composition between stellar populations appears to explain the difference in the slopes of these branches in the two types of clusters. In contrast, in massive stars, the core contraction does not lead to degeneracy but to rapid heating, and the expansion of the initially radiative envelope appears to be so fast as to lead the star hurriedly across the Hertzsprung gap in the Hertzsprung-Russell diagram. Only when the typical red giant state is reached and the envelope has largely become convective does the extraordinary speed of expansion drop.

For stars more massive than about two solar masses, spectroscopic as well as statistical data appear to suggest strongly that mass ejection becomes an essential phenomenon for the evolution beyond the normal red-giant state. It has not been possible this far to follow theoretically these most advanced evolutionary phases.

\section{W H I T E D W A R F S}

Even though the last evolutionary developments of stars can not yet be followed theoretically, it appears highly likely and is substantiated by statistical estimates that the majority of stars end in the white-dwarf state. The internal structure of white dwarfs has long been interpreted in terms of completely degenerate stellar models. The range of radii predicted on this basis agrees satisfactorily with the position of the white dwarfs in the Hertzsprung-Russell diagram.

Recent investigations of the thermal conditions in white dwarfs makes it appear probable that their luminosity represents nothing but a steady loss of residual thermal energy. The cooling times thus computed for the faintest white dwarfs are of the same order as the galactic lifetime.

\section{S U M M A R Y}

Altogether it appears that the most populous types of stars which make up the main features of the Hertzsprung-Russell diagram have by now been fairly securely identified with the broad phases of stellar evolution. It is equally clear, however, that there are still many characteristic stellar types, particularly all around the fringes of the Hertzsprung-Russell diagram, which have as yet completely withstood definite theoretical identification. To these uninterpreted star types belong essentially all stars lighter than a tenth of a solar mass, the carbon stars, the long-period variables, the various types of Cepheids, practically all supergiants, the horizontal branch of globular clusters, Wolf-Rayet stars, and novae and super-novae. There can be little doubt that some of these as yet uninterpreted stellar types will turn out to represent the most advanced evolutionary phases, which up to now we have not understood and which may well become the most exciting part of stellar evolution theory. 